\title{
Review of: "Secondary metabolic profiles and anticancer actions from fruit extracts of immature pomegranates"
}

Maria-João Cebola

Potential competing interests: The author(s) declared that no potential competing interests exist.

The article reports a well-designed and well-analysed study on the waste material originating from the thinning of 'Wonderful' pomegranate cultivars. It specifically looks into the chemical composition of hydro-alcoholic extracts obtained from two types of waste, the baby green and the baby red fruits, and is then followed by studies that ascertain the anticancer properties of the extracts. The authors also put forward some hypotheses that can be followed in the future, on the possible compounds responsible for the anticancer activity found in the extracts. The paper is well written, but there still are some misspellings that the authors ought to correct.

Nowadays, when implementation of the circular economy is of the upmost importance for environmental reasons, this type of work that demonstrates the added value of waste material from agricultural practices is very welcome and very useful to expand the possibilities of real-life application. 